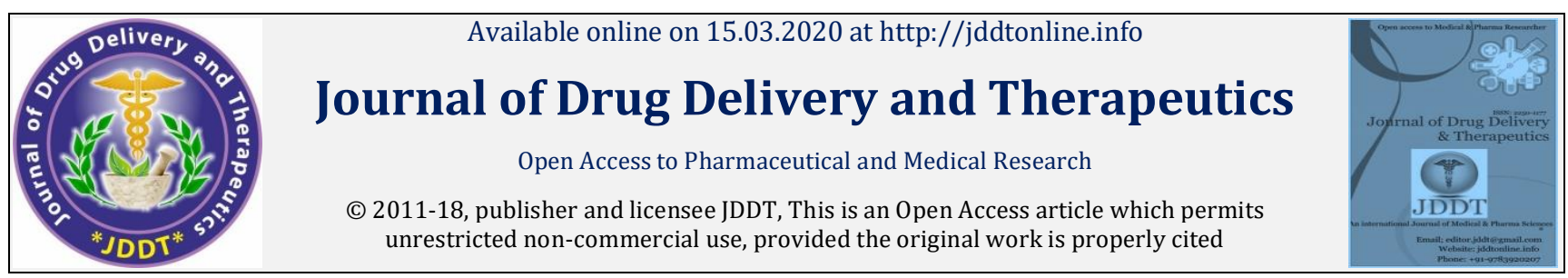

Open Access

Review Article

\title{
The Clinical Aspects of Saroglitazar and its Side Effects
}

\section{Vadlamudi Naga Ratna Sai *1, Sreenivas Pasula*1, Sheelam Sumathi 1, Mondra Sreekanth ${ }^{1}$, A. Srinivas Rao ${ }^{2}$, Beda Durga Prasad ${ }^{3}$}

\author{
1. Pharm D Department of Pharmacy Practice, Bhaskar Pharmacy College, Moinabad, Hyderabad, India \\ 2. Department of Pharmacology, Bhaskar Pharmacy College, Moinabad, Hyderabad, India \\ 3. Department of Chemistry, Bhaskar Pharmacy College, Moinabad, Hyderabad, India
}

\begin{abstract}
The new substance element has been known as novel antidiabetic drug, eg: saroglitazar. saroglitazar is a medication used to treat type-2 diabetes. saroglitazar was known under the exchange name Lipaglyn, created by Zydus cadila. lipaglyn is the first drug approved to treat type2diabetes mellitus by the drug controller general of India in june 2013. Lipaglyn is demonstrated for the patients experiencing diabetes dyslipidaemia. It is given once daily for treatment. Saroglitazar manages the lipid parameters just as glycemic control. [1]
\end{abstract}

Keywords: Anti-diabetic, dual PPAR agonist, glitazar, hypertriglyceridemia, insulin sensitizer, Lipaglyn, AE's (adverse effects).

Article Info: Received 04 Jan 2020; Review Completed 09 Feb 2020; Accepted 20 Feb 2020; Available online 15 March 2020

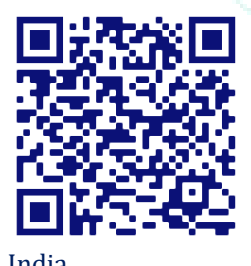

Cite this article as:

Ratna Sai VN, Pasula S, Sumathi S, Sreekanth M, Rao AS, Prasad BD, The Clinical Aspects of Saroglitazar and its Side Effects, Journal of Drug Delivery and Therapeutics. 2020; 10(2):208-212 http://dx.doi.org/10.22270/jddt.v10i2.3941

*Address for Correspondence:

Vadlamudi Naga Ratna Sai, Pharm D Department of Pharmacy Practice, Bhaskar Pharmacy College, Moinabad, Hyderabad, India

\section{INTRODUCTION:}

Dyslipidaemia is considered as one of the major risk factors for cardiovascular diseases (CVD) accounting for $50 \%$ of the myocardial infarction (MI) cases worldwide.[2] A recent epidemiological survey on prevalence of lipid abnormalities of the Indian population

by Indian Council of Medical Research-India Diabetes Study (ICMR-INDIAB), has shown that $79 \%$ of Indian subjects greater than 20 years of age have abnormalities in at least one of the lipid parameters.[3]In this survey, the commonly found lipid abnormality was low-high density lipoprotein cholesterol (low HDL-C) in $72 \%$ subjects followed by high triglycerides(TG) in $29.5 \%$ subjects and then high low-density lipoprotein cholesterol (LDL-C) in $11.8 \%$ subjects.(4) Prevalence of dyslipidaemia is high in India, pharmacological intervention strategies are used to prevent and manage cardiovascular risk factor. Statins the first line treatment for dyslipidaemia and decrease LDL-C levels as well as the danger of cardiovascular occasions in patients with or without cardiovascular disease.[5] Intensive statin treatment was compared with moderatedose statin treatment steadily brings down LDL cholesterol levels and paces of non-lethal cardiovascular events. [6]The extent of CVD chance decrease as an outcome of LDL-C bringing down to the range of $25 \%$ and $35 \% .{ }^{[3]}$ This measurably important but clinically insufficient control of CVD risk is to a limited extent because of a lipid treatment centre around LDL-C alone with a resultant disregard of other significant parts of lipoprotein metabolism. [7] Statin treatment may not eliminate CVD Risk related with low HDL and high triglycerides. [8,9] This review article centres around raised serum triglycerides (TG) and triglyceride rich lipoproteins as potential components limiting further decrease in CVD events in spite of low on-treatment LDL-C. [10]

\section{NEWER CONCEPT —DUAL PPAR $\alpha / \gamma$ AGONIST:}

Peroxisome proliferator-activated receptors (PPARs) are nuclear lipid-activated interpretation factors that regulates the expression of genes to control the lipid and lipoprotein metabolism, glucose homeostasis and inflammatory process. There are 3 PPARs subtypes which are usually assigned PPAR alpha, PPAR gamma and PPAR B/d. PPAR $\alpha$ enactment builds high thickness lipoprotein cholesterol synthesis, stimulates "reverse" cholesterol transport and decreases triglycerides. PPAR activation brings about insulin sensitization and antidiabetic activity. Consolidated medicines with PPAR and an agonist may conceivably improve insulin resistance and ease atherogenic 
dyslipidaemia, while PPAR d properties may prevent the development of overweight which ordinarily goes with "unadulterated" PPAR $\gamma$ ligands. The new generation of double activity PPARs offer a desire for another way to deal with diabetes care tending to glycemia, yet dyslipidaemia and different segments of the metabolic syndrome. [11] Saroglitazar is a novel double PPAR $\alpha / \gamma$ agonist, non-thiazolidinediones (TZD) and non-fibric corrosive subsidiary, with an overwhelming PPAR $\alpha$ agonistic movement. It is a glitazar endorsed by Drug Controller General of India (DCGI) and has been found to have a great pre-clinical and clinical safety profile together with a higher adequacy in enhancing lipid and glycaemic targets. [12,13]

\section{DRUG DESCRIPTION:}

Saroglitazar (trade name Lipaglyn) is a drug to treat diabetes mellitus type 2 and dyslipidaemia. It is approved for use in India by the Drug Controller General of India. [14] Saroglitazar is indicated for the management of diabetic dyslipidaemia and hypertriglyceridemia with type 2 diabetes mellitus which was not improved by statin therapy. In clinical studies, saroglitazar has shown the reduction in triglycerides (TG), LDL cholesterol, VLDL cholesterol, non-HDL cholesterol and an increase in HDL cholesterol a characteristic hallmark of Atherogenic diabetic dyslipidaemia (ADD). It has also shown favourable Anti-diabetic medication property by reducing the fasting plasma glucose and HBA1c in diabetes patients. The recommended dose of saroglitazar is a tablet of $4 \mathrm{mg}$ once a day.

\section{CATEGORY:}

Antidyslipidemic with antidiabetic agent, Peroxisome Proliferator-Activated Receptor (PPAR) Alpha Agonist, Peroxisome Proliferator-Activated Receptor (PPAR) Gamma Agonist. [14]

\section{MECHANISM OF ACTION:}

saroglitazar is a novel first in class drug which acts as a dual PPAR agonist at the subtypes a (alpha) and (gamma) of the peroxisome proliferator-activated receptor (PPAR). Agonist action at PPAR alpha lowers high blood triglycerides, and agonist action on PPAR gamma improves insulin resistance and consequently lowers blood sugar. [15]

\section{IUPAC NAME:}

Alpha-ethoxy-4-(2-(2-methyl-5-(4-(methylithio)phenyl)1H-pyrrol-1-yl)ethoxy)<smiles>CCO[C@@H](Cc1ccc(OCCn2c(C)ccc2-c2ccc(SC)cc2)cc1)C(=O)O</smiles>

CHEMICAL NAME: Benzene propanoic acid.

EMPIRICA FORMULA: C25H29NO4S

MOLECULAR MASS: $900 \mathrm{~g} / \mathrm{mole}$

\section{COMPOSITION}

Each uncoated tablet contains saroglitazar $4 \mathrm{mg}$, excipient quantity sufficient. Inactive ingredients in the tablet are microcrystalline cellulose, lactose, magnesium oxide, povidone, talc, magnesium stearate and colloidal silicon dioxide. [16]

\section{CLINICAL SAFETY / ADVERSE EVENTS WITH SAROGLITAZAR:}

At some stage in the medical trials, all patients on saroglitazar were evaluated for renal, liver, muscle, cardiac functions at baseline, while on therapy and at the completion of study. Saroglitazar become well tolerated over the path of medical trials and no significant changes were determined in these parameters. 2Decho and ECG findings before and after saroglitazar treatment and throughout the observe-up duration did no longer reveal any alteration in cardiac function. Edema, weight advantage, bone modifications, and muscle ache have been additionally no longer said.

The most common adverse effects (AEs $\geq 2$ ) reported were redness, infirmity and febrility that were del icate to moderate in nature and failed to end in conclusion of the drug. Saroglitazar has completed virtually twelve

years of diagnosing and clinical analysis journey with successes while not raising any alarm of toxicity and is approved science Sep 2013 for clinical use in diabetic dyslipidaemia and hypertriglyceridemia not controlled by statins alone. The recommended dose of saroglitazar is 4 mg once daily orally. the Table1 regarding this was given below: $[17,18]$

Table 1: Clinical safety of saroglitazar ${ }^{17,18]}$

\begin{tabular}{|l|l|l|}
\hline Phase 3RCTs & $\begin{array}{l}\text { Saroglitazar } \\
\text { 2mg (\%) }\end{array}$ & $\begin{array}{l}\text { Saroglitazar } \\
\text { 4mg (\%) }\end{array}$ \\
\hline Peripheral edema & 2.4 & 0 \\
\hline Asthma & 2.4 & 7.3 \\
\hline Gastritis & 0 & 4.9 \\
\hline Chest discomfort & 2.4 & 2.4 \\
\hline Dizziness & 2.4 & 2.4 \\
\hline Tremors & 2.4 & 2.4 \\
\hline Pain & 0 & - \\
\hline Pyrexia & - & - \\
\hline Dyspepsia & - & - \\
\hline
\end{tabular}

\section{SIDE EFFECTS:}

Major \& minor side effects for Saroglitazar were: Stomach pain Nausea Vomiting Chest Pain Fever Dizziness. [19]

\section{USES OF SAROGLITAZAR:}

Diabetic Dyslipidaemia: Saroglitazar is used to treat diabetic dyslipidaemia, a condition characterized by abnormal levels of lipids such as triglycerides and cholesterol in the blood of patients suffering from diabetes mellitus, that are not controlled by statin therapy alone. Hypertriglyceridemia with Type-2 Diabetes Mellitus: Saroglitazar is used for the treatment of hyper triglyceridemia, a condition characterized by elevated triglycerides levels in patients suffering from type 2 
diabetes mellitus, that are not controlled by statin therapy alone. [19]

\section{EFFICACY, SAFETY AND TOLERABILITY STUDIES OF SAROGLITAZAR:}

Saroglitazar was planned in the year 2001 after which it was broadly assessed in different preclinical and clinical preliminaries for a long time following which it was at last endorsed in 2013 for showcasing in India in patients experiencing diabetic dyslipidaemia and hypertriglyceridemia in type 2 diabetic patients not constrained by statins alone.

\section{EFFICACY:}

Being a double PPAR agonist, Saroglitazar (Lipaglyn) helps in controlling blood glucose and Lipid parameters particularly high triglycerides and high non-HDLCholesterol. [20] Lipaglyn successfully diminishes triglycerides and non-HDL-C and controls high glucose, a regular circumstance in Insulin Resistance condition. [21,22]

\section{SAFETY AND TOLERABILITY:}

Saroglitazar has not shown any of the unfavourable impacts like weight increase and edema that are normally related to comparable atoms like the glitazone class of drugs. [23] Because it is an insulin sensitizer, Saroglitazar (Lipaglyn) has less potential for hypoglycaemia. No major serious adrs have been accounted for; nonetheless, long haul cardiovascular security has not been set up. [24] Saroglitazar was protected and all around endured up to a 128 MG oral portion during the single-climbing portion and furthermore during the investigation of the impacts of nourishment and sex. There was no genuine $\mathrm{AE}$ seen during the examination in any treatment arm.

A sum of 22 AEs in 11 subjects were accounted for during the investigation, included rash/tingling, stomach torment, queasiness, hack, cold, migraine, spinal pain, body torment, calf torment, fever, disquietude, happiness, dyspepsia and looseness of the bowels; in any case, they were gentle to direct in force. None of the AEs was treatment new, and none required any treatment for goals. There was no clinically important pattern or change saw in clinical research facility, urinalysis or ECG discoveries in the fake treatment or treatment arms during the investigation. There was no reliable example or portion reliance saw in the AEs. [25]

\section{PHARMACOKINETICS:}

It is administered orally, rapidly absorbed, good oral bioavailability of $40 \%, \mathrm{t} 1 / 2$ of about $3-4$ hrs. saroglitazar was metabolized in to 3 minor oxidative metabolites and it was predominantly eliminated unchanged by the non-renal route (Hepatobiliary route).

\section{PHARMACODYNAMICS:}

PPAR an activation by saroglitazar will increase the internal organ (LIVER) chemical reaction (OXIDATION) of fatty acids (FA) and reduces the synthesis and secretion of Triglycerides (TG). This, in turn, will increase diversion of Fatty acids from peripheral tissues (Eg, Skeletal muscle and fat tissue) to the liver, thereby decreasing both fatty acid synthesis and delivery of Triglycerides to peripheral tissues. Saroglitazar additionally causes increased lipolysis and elimination of Triglyceride-rich particles from plasma by activating Lipoprotein Lipase (LPL) and reducing production of Apo C-III, an inhibitor of LPL activity. Saroglitazar was additionally found to reduce plasma LDLC. PPAR activation by saroglitazar additionally induces an increase in the synthesis of apolipoproteins A-I and A-II, and HDL-C.(27) Although saroglitazar is predominantly a PPAR $\alpha$ agonist, it additionally causes activation of PPAR $\gamma$ and regulates the transcription of insulin-responsive genes concerned within the control of glucose production, transport, and utilization. Saroglitazar will increase the expression of diverse PPAR $\gamma$ responsive genes concerned in carbohydrate and lipid metabolism, together with adiponectin, adipocyte fatty acid-binding protein, LPL, fatty acid transport protein, and fatty acid translocase (CD36). By increasing the expression of these genes, saroglitazar decreases the postprandial rise of plasma FFA, improves postabsorptive, insulin-mediated suppression of hepatic glucose output, reduces the metabolic burden on liver and muscle, and promotes glucose utilization. Robust antidiabetic drug and insulin-sensitizing effects of saroglitazar were observed in preclinical models, within which hyperglycemia and/or impaired glucose tolerance may be a consequence of insulin resistance in target tissues. [27] As mentioned, saroglitazar is preponderantly a PPAR $\alpha$ agonist, with modest PPAR $\gamma$ agonist actions. This is in contrast to previously developed dual PPAR $\alpha / \gamma$ agonists, like muraglitazar, aleglitazar, and tesaglitazar, that had either predominantly PPAR $\gamma$ agonistic activity or equivocal agonistic activity on each PPAR $\alpha$ and PPAR $\gamma$ receptors.

\section{CELL LINES AND TREATMENT:}

HepG2 cells were maintained in DMEM supplemented with 10 percent FBS and a $100 \mathrm{U} / \mathrm{mL}$ penicillin/streptomycin (Invitrogen Carlsbad, CA, USA). Human hepatic stellate LX2 cells were maintained in DMEM with five percent FBS. The cells were maintained at $37^{\circ} \mathrm{C}$ and five percent carbon dioxide. Palmitate (PA, Sigma- Aldrich) was conjugated to BSA before use in serum- starved cells. For coculture experiments, HepG2 and LX2 were plated on inserts (0.4$\mu \mathrm{m}$ pore size, Corning, USA) and on the bottom plate at a ratio of 5:1 severally. the 2 different cell populations were cocultured for seventy-two hours and harvested for downstream experiments. Cells were treated with saroglitazar, fenofibrate, pioglitazone (all treated at $10 \mu \mathrm{M}$ concentration) and PA $(0.75 \mathrm{mM})$ for sixteen hours. ribonucleic acid and protein were isolated. The relative gene expression of fibrosis markers was quantified by qPCR from the mRNA obtained from HepG2- LX2 cells cocultured and 18S rRNA was used as housekeeping gene. Isolated proteins were subjected to immunoblotting with antibodies specific for anti- pNFkB and total NFkB (Cell sign Technology; dilution 1:1000). Densitometric. For Mito stress assay and assessment of reactive oxygen species (ROS), HepG2 cells were plated at a density of 35,000 cells/well in XFe24- well plates (Seahorse Biosciences). The oxygen consumption rate (OCR) was measured according to the manufacturers protocol. Oligomycin (2M), FCCP (2M), rotenone (1M) and antimycin A (1M) were used as inhibitors of various mitochondrial metabolic respiratory complexes in all wells. For the assessment of ROS generation, HepG2 cells were stained with dichlorodihydrofluorescein diacetate (DCFDA) (5 M) followed by washing in PBS. The harvested cells were suspended in PBS for detection by flow cytometry (BD Biosciences). For assessment of stellate cell activation, LX2 cells were fixed in $3.7 \%$ paraformaldehyde. when PBS washing, cells were stained with phalloidin (1:500) and subsequently stained with DAPI for forty-five minutes followed by observation via an Olymps Fluoview Confocal microscopy (Tokyo, Japan). [28] 


\section{POST-MARKETING SURVEILLANCE EVIDENCES OF EFFICACY AND SAFETY (PHASE IV STUDIES):}

An observational, multicentre, post-marketing study (phase IV) involving 2804 subjects by Shetty R et al [29] revealed that saroglitazar additionally to oral antidiabetic drug (at outpatient clinics) showed significant improvement in all lipid and glycemic parameters at three months follow-up. The mean baseline TG was $312.3 \mathrm{mg} / \mathrm{dL}$ vs. $188.7 \mathrm{mg} / \mathrm{dL}$ at three months follow-up, a major reduction of $35.8 \%$ (mean of \% change from baseline). Non-HDLC levels additionally reported a major $23.4 \%$ mean reduction at three months follow-up. A statistically significant improvement in all other lipid parameters was also noted (a mean reduction of $16.4 \%$ in LDL-C levels, $31.5 \%$ in VLDL-C levels, $19 \%$ in total cholesterol levels and mean increase of $7.3 \%$ in HDL-C levels). Analysis of glycaemic parameters revealed a statistically significant $0.9 \%$ absolute reduction in glycated haemoglobin (HbA1c) from baseline value of $8.3 \%$ to $7.4 \%$ at three-month follow-up. there have been no serious adverse events or weight gain rumoured in study. Saroglitazar has been given at various International platforms, recently two years post-marketing surveillance data of saroglitazar was given at American Diabetic Association (ADA) conference, 2016. According to the data presented in ADA 2016, two years use of saroglitazar in diabetic patients with high triglycerides showed a significant $41 \%$ reduction in TGs. Other lipid parameters like non-HDL-C and LDL-C also reduced significantly by $28 \%$ and $12 \%$ respectively. There was significant improvement in glycaemic parameters as well (HbA1c reduced by $0.7 \%$ ) and it was concluded that two years treatment with saroglitazar is effective, safe, well tolerated and isn't associated with edema, weight gain or any other serious adverse event. [30]

\section{CONCLUSION}

(31) Because of the various ethnicity of Indians, atherogenic dyslipidaemia is highly prevalent within the Indian population which comprises of high concentrations of TGs, low concentrations of HDL-C, and moderate increase in LDL-C, with an increased concentration of small, dense, and potentially more atherogenic particles. There is a growing support for unadjusted elevated triglyceride levels as an independent CVD risk factor and management of hypertriglyceridemia has currently become equally very important in reducing the CVD events. The medication out there for this current management of hypertriglyceridemia in T2DM have their own specific limitations. Therefore, within the management of $\mathrm{AD}$, several unmet needs still exist and a significant residual CVD risk prevails despite the current optimal therapy. A dual PPAR $\alpha / \gamma$ agonist (saroglitazar) by reducing both the TG \&insulin resistance with a positive safety profile might provide anoptimal lipid and glycaemic targets. Saroglitazar (a dual PPAR $\alpha / \gamma$ agonist) is the first glitazar class compound that has been approved as a therapeutic agent for the excellent management of atherogenic diabetic dyslipidaemia or hypertriglyceridemia in diabetes not controlled by statins alone. The broad range of lipid improvements associated with saroglitazar addresses the pattern of dyslipidaemia commonly seen in Indians. Additionally, by improving the insulin sensitivity, saroglitazar provides an optimal glycaemic control also. It is novel because it is the first in its class approved anywhere in the world, with superior efficacy in reducing TG and non-HDL-C and a dual action of controlling both dyslipidaemia and hyperglycaemia. However, in spite of proven therapeutic advantages, large outcome study of saroglitazar showing long-term therapeutic efficacy and safety is needed.

\section{ACKNOWLEDGEMENT:}

We thank Dr. Sreenivas Pasula (PharmD (PhD), Department of pharmacy, Bhaskar Pharmacy College) Who guided me through this article. We thank Dr. A. Srinivas Rao (principal, Bhaskar Pharmacy College) for providing support, computer laboratory facility, online accessibility of articles and other resources. Finally, we thank the anonymous referees for their useful suggestions.

\section{REFERENCES}

[1]: Agarwal R. The First approved agent in the Glitazar's Class: Saroglitazar, PubMed.gov,. 2014; 5(2)

[2]: Yusuf S, Hawken S, Ounpuu S, et al. Effect of potentially modifiable risk factors associated with myocardial infarction in 52 countries (the INTERHEART study). Lancet. 2004; 364: 937-952.

[3]: La Rosa JC, He J, Vupputuri S. Effect of statins on risk of coronary disease: A meta-analysis of randomized controlled trials JAMA. 1999; 282: 2340-2346.

[4]: Joshi SR, Anjana RM, Deepa M, et al. Prevalence of dyslipidemia in urban and rural India: The ICMR-INDIAB Study. PLOS ONE. 2014; 9(5): 96808.

[5]: Baigent C, Keech A, Kearney PM, et al. Cholesterol treatment trialists (CTT) collaborators. Efficacy and safety of cholesterol lowering treatment: Prospective meta-analysis of data from 90,056 participants in 14 randomized trials of statins. Lancet. 2005; 366: 1267-1278.

[6]: Cannon CP, Steinberg BA, Murphy SA, Mega JL, Braunwald E. Meta-analysis of cardiovascular outcomes trials comparing intensive versus moderate statin therapy. Journal of the American College of Cardiology. 2006; 483: 438-445.

[7]: Cannon $\mathrm{CP}$, Braunwald $\mathrm{E}, \mathrm{McCabe} \mathrm{CH}$, et al. Intensive versus moderate lipid lowering with statins after acute coronary syndromes. N Engl J Med. 2004; 350: 1495-1504.

[8]: Adapted from HPS Collaborative Group. MRC/BHF Heart Protection Study of antioxidant vitamin supplementation in 20 536 high-risk individuals: A randomized placebo-controlled trial. Lancet. 2002; 360: 7-22.

[9]: Sacks FM, Tonkin AM, Shepherd J, et al. Effect of pravastatin on coronary disease events in subgroups defined by coronary risk factors, the prospective pravastatin pooling project. Circulation 2000; 102: 1893-900. doi: 10.1161/01.CIR.102.16.1893

[10]: Miller M. Current perspectives on the management of hypertriglyceridemia. Am Heart J. 2000; 140: 232-240.

[11]. Fredenrich A, Grimaldi PA. PPAR delta. An uncompletely known nuclear receptor. Diabetes Metab. 2005; 31: 23-27.

12. Munigoti SP, Harinarayan CV. Role of glitazars in atherogenic dyslipidemia and diabetes: Two birds with one stone. Indian Endocrinol Metab. 2014; 18(3): 283-287.

[13]: Jani H, Kansagra K, Jain MR, Patel H. Pharmacokinetics, safety, and tolerability of saroglitazar (ZYH1), a predominantly PPAR $\alpha$ agonist with moderate PPAR $\gamma$ agonist activity in healthy human subjects. Clin Drug Investig. 2013; 33(11): 809-816.

[14]: Zydus Group launches new diabetic drug "The Times of India. Jun 6, 2013.

[15]: Lipaglyn (Saroglitazar) for Treating Hypertriglyceridemia in Type II Diabetes, India". Drug Development and Technology. 2014; 8:132-141. 8.

[16]: [Internet]. Lipaglyn.com. 2018 [cited 19 September 2018].

[17]: Pai V, Paneerselvam A, Mukhopadhyay S, et al. A Multicenter, prospective, randomized, double-blind study to evaluate the safety and efficacy of saroglitazar 2 and $4 \mathrm{mg}$ compared to pioglitazone $45 \mathrm{mg}$ in diabetic dyslipidemia (PRESS V). J Diabetes Sci Technol 2014; 8(1):132-141. 
[19]: Sosale et al, Saroglitazar for the treatment of hypertriglyceridemia in patients with type 2 diabetes: current evidence, Dove press 2015:8 189-196.

[18]: Jani R, Pai V, Jha $P$, et al. A multicenter, prospective, randomized, double-blind study to evaluate the safety and efficacy of saroglitazar 2 and $4 \mathrm{mg}$ compared with placebo in type 2 diabetes mellitus patients having hypertriglyceridemia not controlled with atorvastatin therapy (PRESS VI). Diabetes Technology \& Therapeutics. 2014; 16(2): 63-71.

[20]: Manoria, PC, Chopra, HK, Parashar, SK, Dutta, AL, Pinto, B, Mullasari, A, Prajapati, S. "The nuances of atherogenic dyslipidaemia in diabetes: focus on triglycerides and current management strategies “. Indian Heart Journal. 65 (6): 68390.

[21]: Observational study of Effects of Saroglitazar on Glycaemic and Lipid Parameters on Indian Patients with Type 2 Diabetes “. SCIENTIFIC REPORTS.

[22]: Ramakrishnan, S. "-From 'Make in India 'to Made in India ': the saroglitazar story ". Indian Heart Journal. 67 (1): 8-10.

[23]: Shetty, SR, Kumar, S, Mathur, RP, Sharma, KH, Jaiswal, AD. "Observational study to evaluate the safety and efficacy of saroglitazar in Indian diabetic dyslipidaemia patients ". Indian Heart Journal. 67 (1): 23-6.

[24]: Munigoti, Srinivasa P, Harinarayan, CV. "Role of Glitazars in atherogenic dyslipidaemia and diabetes: Two birds with one stone? ". Indian Journal of Endocrinology and Metabolism. 2014; 18(3): 283-7.

[25]: Jani RH, Kansagra KK, Patel H. Pharmacokinetics, Safety and Tolerability of Saroglitazar (ZYH1), a Predominantly PPAR alpha Agonist with Moderate PPAR gamma Agonist Activity in Healthy Human Subjects. Clinical Drug Investigation, 2013; 33(11): 809816

[26]: Sonu S. Biliary excretion of ZYH1 in wistar rats. Ahmedabad: Cadila Healthcare Ltd.; 2004.

[27]: Lipaglyn (Saroglitazar) [prescribing information]. Ahmedabad, India: Cadila Healthcare; 2013.

[28]: Jain MR et al, Dual PPAR Alpha/Gamma agonist saroglitazar improve liver histopathology and biochemistry in experimental NASH models. Liver Int. 2018;38(6):1084-94.

[29]: Shetty S, Kumar S, Mathur R, Sharma K, Jaiswal A. Observational study to evaluate the safety and efficacy of saroglitazar in Indian diabetic dyslipidemia patients. Indian Heart Journal. 2015; 67(1): 23-26.

[30]: Joshi S, Sound R, Saboo B. Efficacy and Safety of Saroglitazar in Indian Diabetics-two year data. Adapted from ADA 2016;

31. Bhatia V, Arora P, Kaur G, Kaul U. Saroglitazar: A New Drug to Treat. Res Open J. 2016; 4(1): 12-17. doi: 10.17140/HROJ-4-135 